\title{
Tetrandrine inhibits glioma stem-like cells by repressing $\beta$-catenin expression
}

\author{
YONG ZHANG $^{1,2^{*}}$, YU-LIN WEN ${ }^{1,2^{*}}$, JI-WEI MA $^{1,2,5^{*}}$, JIE-CHENG YE $^{1,2}$, XIAO WANG $^{3}$, \\ JIAN-XIAN HUANG ${ }^{1,2}$, CHAO-YUE MENG ${ }^{1,2}$, XIAO-ZE XU ${ }^{1,2}$, SHAO-XIANG WANG ${ }^{4}$ and XUE-YUN ZHONG ${ }^{1,2}$ \\ ${ }^{1}$ Department of Pathology, Medical School of Jinan University, Guangzhou, Guangdong 510632; \\ ${ }^{2}$ Guangdong Province Key Laboratory of Molecule Immunology and Antibody Engineering, ${ }^{3}$ Guangzhou Jinan Biomedicine \\ Research and Development Center, National Engineering Research Center of Genetic Medicine, Jinan University, Guangzhou, \\ Guangdong 510632; ${ }^{4}$ School of Medicine, Shenzhen University, Shenzhen, Guangdong 518060; \\ ${ }^{5}$ Department of Pathology, Shandong Provincial Hospital Affiliated to Shandong University, Jinan, Shandong 250021, P.R. China
}

Received August 7, 2016; Accepted November 7, 2016

DOI: 10.3892/ijo.2016.3780

\begin{abstract}
Cancer stem cells (CSCs) in glioma are often responsible for relapse and resistance to therapy. The purpose of the present study was to confirm the self-renewal and migration inhibitory effects of tetrandrine (Tet), which is a compound extracted from the dried root of Stephania tetrandra S. Moore, toward glioma stem-like cells (GSLCs) and to examine the associated molecular mechanisms. Using a neurosphere culture technique, we enriched the GSLC population from the human glioblastoma cell lines U87 and U251. Cells were analyzed using Cell Counting kit-8 (CCK-8), western blotting, flow cytometry, Transwell assay and immunofluorescence staining. GSLCs displayed properties of neural stem cells, including elevated expression of the cancer stem cell marker ALDH1 and $\beta$-catenin. We found that Tet treatment decreased sphere formation in GSLCs in a dosedependent manner using tumor spheroid formation assay. The GSK $3 \beta$ inhibitor BIO maintained sphere formation and migration capacity in GSLCs, whereas the $\beta$-catenin/TCF transcription inhibitor ICG-001 decreased sphere formation and the migration capacity of GSLCs. The proportion of
\end{abstract}

Correspondence to: Dr Xue-Yun Zhong, Department of Pathology, Medical School of Jinan University, Guangzhou, Guangdong 510632, P.R. China

E-mail: tzxy@jnu.edu.cn

Dr Shao-Xiang Wang, School of Medicine, Shenzhen University, Shenzhen, Guangdong 518060, P.R. China

E-mail: hackerwsx@163.com

*Contributed equally

Abbreviations: CSCs, cancer stem cells; GSLCs, glioma stem-like cells; Tet, tetrandrine; TdT, terminal deoxynucleotidyl transferase

Key words: tetrandrine, glioma, Wnt/ $\beta$-catenin, nuclear, translocation, stem cell, neurosphere culture apoptotic GSLCs also increased in response to ICG-001 treatment. These results indicate that $\beta$-catenin activity is vital in maintaining neural stem cell traits of GSLCs. Tet inhibits cell viability, neurosphere formation and migration of GSLCs in vitro. Importantly, Tet treatment significantly repressed the nuclear translocation and expression of $\beta$-catenin and induced apoptosis in GSLCs, as indicated in part by the upregulation of Bax, the cleavage of PARP and the downregulation of Bcl-2. The present study demonstrates that the inhibition of $\beta$-catenin in CSCs by Tet could be an effective strategy for the treatment of glioma.

\section{Introduction}

Glioma is the most common type of primary malignant tumor of the central nervous system (1). Despite the current standard of care for glioma patients, which involves surgery, radiotherapy and chemotherapy, the prognosis of glioma patients remains poor. The median overall survival for World Health Organization (WHO) grade IV glioblastoma patients is only 14.6 months (2). Therefore, it is essential to improve the treatments for glioma patients.

A previous report shows that the prognosis and malignant degree of gliomas are correlated with the presence of glioma stem-like cells (GSLCs) (3). Glioma stem-like cells are a small cellular subpopulation that exhibits the capacity for self-renewal and differentiation. Glioma stem-like cells are also thought to contribute to glioma resistance to conventional radiotherapy and chemotherapy. The expression of cancer stem cell-related markers in gliomas is often associated with the survival of glioma stem cells (4). Therefore, enriching and identifying glioma stem-like cells is essential for the development of novel therapeutic approaches targeting glioma stem cells.

$\beta$-catenin is a crucial factor involved in cancer stem cell self-renewal. After entering the nucleus and binding with the TCF/LEF family transcription factors, the activation of $\beta$-catenin induces the expression of target genes to maintain cancer stem cell survival (5). The expression of $\beta$-catenin 
affects the mRNA stability of the cancer stem growth factor IL6 (6). In Wnt/ $\beta$-catenin signaling, GSK3 $\beta$ forms a multimeric complex with $\beta$-catenin, AXIN1 and APC. The destruction of the inactivated complex results in the cytoplasmic accumulation and nuclear translocation of $\beta$-catenin, where it associates with TCF/LEF and alters the transcriptional state of the cell. Recent studies have shown that the activation of $\mathrm{Wnt} / \beta$-catenin signaling helps maintain the glioma stem-like phenotype $(7,8)$. Wnt/ $\beta$-catenin signaling also plays an important role in regulating colorectal cancer stem cells (9). Thus, the inhibition of Wnt/ $\beta$-catenin signaling in GSLCs could be a promising strategy for glioma therapy.

Tetrandrine (Tet) is a compound extracted from the dried root of Stephania tetrandra S. Moore. Tet has been widely used due to its anti-inflammatory, immunosuppressive and anti-hypertensive effects (10). Recent studies have shown that Tet has antitumor activity for several cancer cells, including neuroblastoma, breast, lung, colon and prostate cancer cells, and the anticancer effects of Tet could be mediated in part by the inactivation of Wnt/ $\beta$-catenin signal transduction (11-15). However, neither the effects of Tet in GSLCs nor its mechanism of action have been evaluated. In this study, we identified and enriched GSLCs from the U87 and U251 cell lines. Furthermore, we evaluated the effects of Tet on GSLCs, and we found that Tet inhibits GSLCs by repressing the expression of $\beta$-catenin and preventing its nuclear translocation.

\section{Materials and methods}

Patients and tissue collection. Eighty-eight patients with gliomas were included in the present study. All patients underwent surgery at the Second People's Hospital of Shenzhen (Shenzhen, China) between October 2004 and August 2010. This study cohort consisted of 51 males and 37 females, with a median age of 36 years (range, 3-72 years). All patients underwent magnetic resonance imaging (MRI) a few days before surgery and within $72 \mathrm{~h}$ after surgery. The extent of tumor resection was determined using postoperative MRI scans. Surgical resection was defined as macroscopic total resection, partial resection, or biopsy, as appropriate. To confirm the diagnosis, two neuropathologists independently evaluated the tumor samples according to the WHO criteria: 47 patients presented with stage I-II disease and 41 patients presented with stage III-IV disease.

Cell lines and cultures. Human glioblastoma cell lines U87 and U251 were obtained from the Shanghai Institute of Cell Biology, Chinese Academy of Sciences (Shanghai, China). All cells were cultured in Dulbecco's modified Eagle's medium (DMEM; Gibco-Life Technologies, Paisley, UK) supplemented with $10 \%$ fetal bovine serum (FBS), $100 \mathrm{IU} / \mathrm{ml}$ penicillin and $100 \mathrm{mg} / \mathrm{ml}$ streptomycin. Cells were maintained in an atmosphere of $5 \% \mathrm{CO}_{2}$ at $37^{\circ} \mathrm{C}$.

Neurosphere culture. Glioblastoma cells were plated in a $60-\mathrm{mm}$ dish $(5,000$ cells $/ \mathrm{ml})$ in neurobasal medium containing B27 (Invitrogen, San Diego, CA, USA), 20 ng/ml human basic fibroblast growth factor (Sigma-Aldrich, Taufkirchen, Germany) and $20 \mathrm{ng} / \mathrm{ml}$ epidermal growth factor (Invitrogen, Carlsbad, CA, USA) for 7 days. Spheroids were collected after 7 days and were dissociated with Accutase (a mixture of enzymes with proteolytic, collagenolytic and DNase activity; Invitrogen). The cells obtained from dissociation were filtered through a $40-\mu \mathrm{m}$ cell strainer. Then, the dissociated cells were plated in a $60-\mathrm{mm}$ dish and were maintained in neurobasal medium to enrich for GSLCs. Subsequently, the protein expression levels of stem cell markers were evaluated by western blotting and immunofluorescence staining.

Cytotoxicity assays with Cell Counting kit-8 (CCK-8). The cytotoxic effect of Tet (Sigma-Aldrich, St. Louis, MO, USA) was measured using the CCK-8 assay (Dojindo Laboratories, Kumamoto, Japan). GSLCs were dissociated into single cells, which were seeded into 96 -well plates at a density of $5 \times 10^{3}$ cells/well. Various doses of Tet were added to each well $24 \mathrm{~h}$ after seeding. Spheroids in each well were photographed after treatment by microscopy. The optical density at $450 \mathrm{~nm}$ was measured using a microplate reader (BioTek Instruments, Inc., Winooski, VT, USA). The $\mathrm{IC}_{50}$ value, determined by the relative absorbance of CCK-8, was assessed using probit regression analysis in the SPSS 13.0 statistical software package. The half maximal inhibitory concentration $\left(\mathrm{IC}_{50}\right)$ was calculated using SPSS 13.0 statistical software.

Tumor spheroid formation assay. To assess the sphere-forming capability of GSLCs in vitro, spheroid cells were dissociated into single cells and then were plated in 96-well plates at a density of $1 \times 10^{3}$ cells $/ \mathrm{ml}$ in an atmosphere of $5 \% \mathrm{CO}_{2}$ at $37^{\circ} \mathrm{C}$. Various doses of Tet were added to each well $24 \mathrm{~h}$ after seeding. Spheroids $>0.2 \mathrm{~mm}$ in diameter in each well were photographed under a microscope after 7 days of incubation.

Transwell migration assay. For the Transwell migration assays, GSLCs were dissociated into single cells, after which $5 \times 10^{3}$ cells $/ \mathrm{ml}$ were plated in the top Transwell chamber $(6.5 \mathrm{~mm}$ diameter, $8.0 \mu \mathrm{m}$ pore size polycarbonate filters; Corning Incorporated, Corning, NY, USA) and were allowed to migrate toward $600 \mu \mathrm{l}$ of serum-containing medium in the lower Transwell chamber. After $24 \mathrm{~h}$, the cells were fixed with methanol and stained with $0.1 \%$ crystal violet $(2 \mathrm{mg} / \mathrm{ml})$. The number of cells that migrated through the membrane was counted in five randomly selected fields under a light microscope.

FACS analysis. Apoptosis was measured using an Annexin V-FITC/PI apoptosis detection kit (BD Biosciences, San Jose, CA, USA). GSLCs were dissociated into single cells, after which the cells were treated for the indicated amount of time and then harvested and washed twice with cold phosphate-buffered saline (PBS). Then, cells were re-suspended in $100 \mu \mathrm{l}$ of binding buffer $\left(1 \times 10^{5}\right.$ cells), after which the cells were stained with $5 \mu \mathrm{l}$ of Annexin V-FITC and $10 \mu \mathrm{l}$ of PI for $30 \mathrm{~min}$ at room temperature in the dark. The percentage of apoptotic cells was determined by flow cytometry (Beckman Coulter, Brea, CA, USA).

Western blotting. Protein expression levels were determined by western blotting. In brief, the cells were lysed for $30 \mathrm{~min}$ on ice in $300 \mu \mathrm{l}$ of radioimmunoprecipitation assay (RIPA) lysis buffer. The lysates were subjected to sodium dodecyl sulfate (SDS)-polyacrylamide gel electrophoresis (PAGE) separation, 


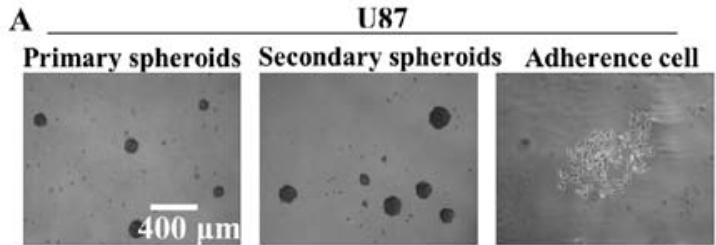

U251
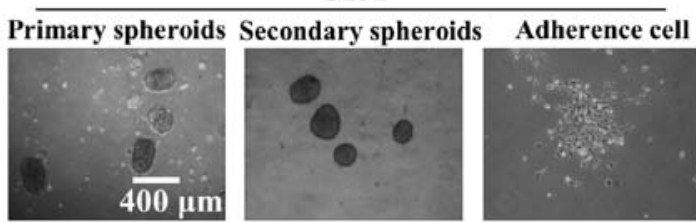

C

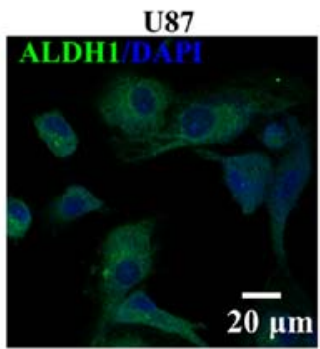

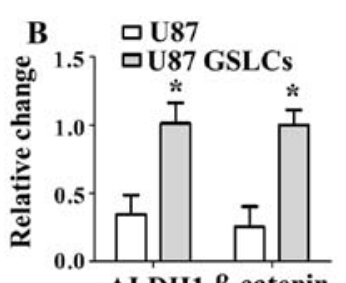

ALDH1 $\beta$-catenin

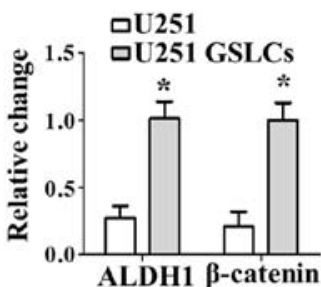

U251 U251 GSLCs

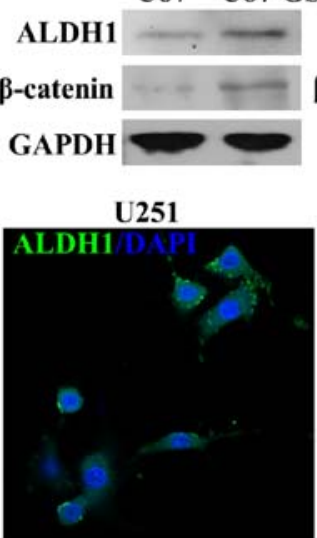

ALDH1

$\beta$-catenin

GAPDH

U251 GSLCs

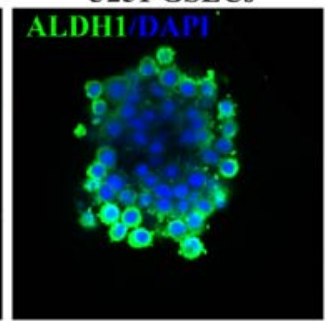

Figure 1. CSLCs from the U87 and U251 cell lines exhibit characteristics of CSCs as shown by increased expression of ALDH1. (A) Primary spheroids were developed from a monolayer of U87 and U251 cells after culture for 7 days in serum-free DMEM/F12 supplemented with B-27, EGF and bFGF. Primary spheroids were dissociated with Accutase, filtered through a $40-\mu \mathrm{m}$ cell strainer and seeded in serum-free medium to obtain secondary spheroids. Secondary spheroids present as cancer stem-like cells and exhibit the properties of neural stem cells. Secondary spheroids attached to the plate and proliferated after 3 days of culture in DMEM/F12 supplemented with 10\% FBS. Scale bars, $400 \mu \mathrm{m}$. (B) Protein expression levels of ALDH1 were detected by western blotting and were normalized to GAPDH in U87, U251 and GSLCs. Data represent the means \pm SD. ${ }^{*} \mathrm{P}<0.05$ different from the respective controls. (C) The expression levels of ALDH1 in U87, U251 and GSLCs were observed by immunofluorescence staining. Blue fluorescence represents DAPI, and green fluorescence represents ALDH1. Scale bars, $20 \mu \mathrm{m}$.

after which the proteins were transferred onto polyvinylidene fluoride (PVDF) membranes (Roche Diagnostics GmbH, Penzberg, Germany). The membranes were blocked with $5 \%$ fat-free milk and were incubated overnight at $4^{\circ} \mathrm{C}$ with primary antibodies against ALDH1 (Abcam, Cambridge, UK), GAPDH, $\beta$-catenin, GSK3 $\beta$, Bax, Bcl-2 and cleaved PARP, as indicated (Cell Signaling Technology, Danvers, MA, USA). After five washes of $10 \mathrm{~min}$ each in TBST, the membranes were incubated with horseradish peroxidase (HRP)-conjugated secondary antibodies (1:5,000; Cell Signaling Technology) for $1 \mathrm{~h}$. Immunoreactive bands were visualized using an enhanced chemiluminescence assay (Pierce, Rockford, IL, USA).

Immunofluorescence staining. GSLCs were plated on polyL-lysine-coated coverslips (Sigma-Aldrich) and incubated overnight at $37^{\circ} \mathrm{C}$, after which the cells were treated with Tet, BIO (Selleck Chemicals, Houston TX, USA) or ICG-001 (Selleck Chemicals). After treatment, the cells were rinsed with PBS and fixed in $3.7 \%$ paraformaldehyde for $15 \mathrm{~min}$. Cells were then washed in PBS three times for $5 \mathrm{~min}$, and $0.2 \%$ Triton X-100 was added to the cells. Cells were blocked in 5\% bovine serum albumin for 60 min after three washes in PBS, and then the cells were incubated with anti- $\beta$-catenin antibody (1:100), anti-rabbit-FITC secondary antibody (1:1,000; Cell Signaling Technology), TUNEL reaction mixture containing a nucleotide mixture and terminal deoxynucleotidyl transferase (TdT) (In Situ Cell Death Detection kit; Roche Diagnostics $\mathrm{GmbH}$ ), and 4',6-diamidino-2-phenylindole (DAPI; Cell
Signaling Technology). Finally, the slides were mounted and examined by laser scanning confocal microscopy (LSM).

Immunohistochemical analysis. We prepared slides using 2-mm-thick sections of paraffin-embedded specimens. The slides were baked at $60^{\circ} \mathrm{C}$ for $2 \mathrm{~h}$, deparaffinized in xylene, rehydrated in decreasing concentrations of ethanol and rinsed in PBS. The slides were then microwaved with a $10 \mathrm{mmol} / \mathrm{l}$ citrate buffer (pH 6.0; Maixin Bio, Fuzhou, China) on the 'high' setting for $5 \mathrm{~min}$ and on the 'mid-high' setting for 10 min. The Ultra-Sensitive ${ }^{\mathrm{TM}}$ SP kit (Maixin Bio) was used to incubate the slides with hydrogen peroxide and normal serum for $10 \mathrm{~min}$ each. Next, the slides were incubated overnight with rabbit anti-human monoclonal antibodies to $\beta$-catenin (diluted 1:100; Cell Signaling Technology) at $4^{\circ} \mathrm{C}$. The slides were then processed with the Ultra-Sensitive ${ }^{\mathrm{TM}} \mathrm{SP}$ kit for $30 \mathrm{~min}$ at room temperature, followed by development with diaminobenzidine (DAB) for visualization. Negative controls were included by substituting the primary antibodies with non-immune serum.

Statistical analysis. The data are expressed as the means and standard deviations (SD). The $\chi^{2}$ test was used to analyze the correlations between the clinicopathological features and the $\beta$-catenin expression. Statistical analysis of the remaining data was conducted using a one-way analysis of variance (ANOVA) in the statistical package SPSS 13.0 (SPSS, Inc., Chicago, IL, USA). $\mathrm{P}<0.05$ was considered statistically significant. 

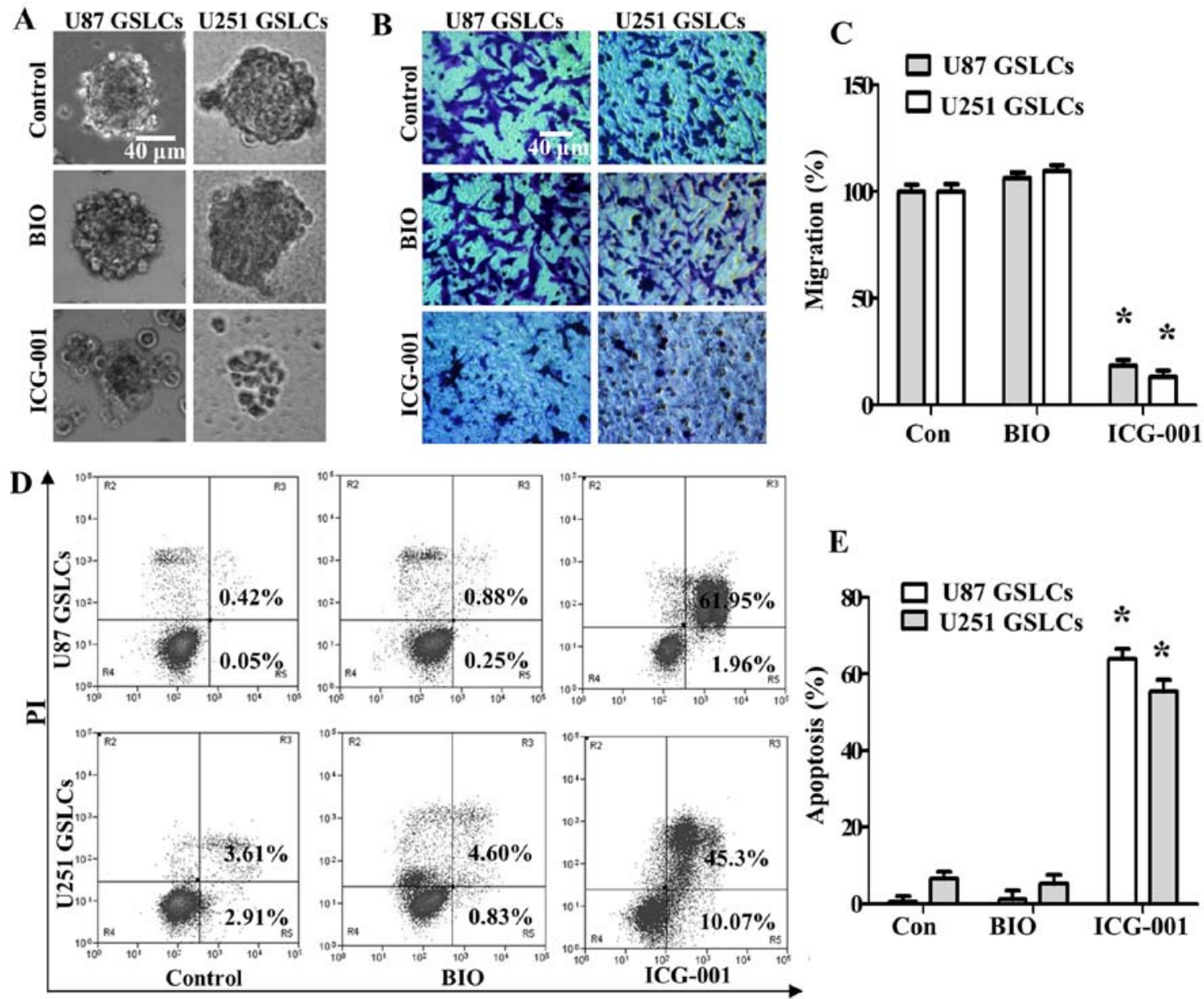

$\mathbf{E}$

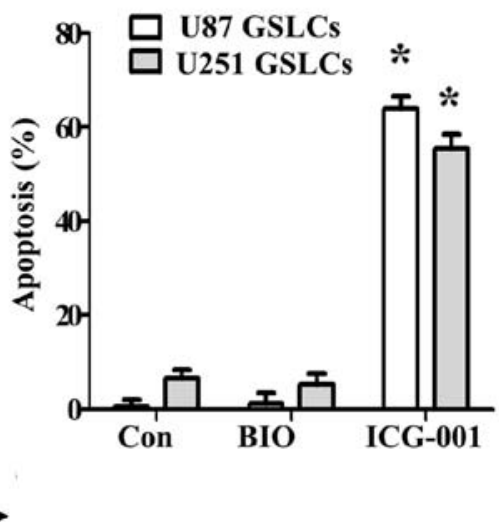

Annexin V

Figure 2. Wnt/ $\beta$-catenin signaling is vital to maintain neural stem cell properties and the survival of GSLCs. (A) GSLCs were dissociated and seeded in suspension culture, after which they were treated with $5 \mu \mathrm{M}$ BIO or $10 \mu \mathrm{M}$ ICG-001 for 5 days in 6-well plates. Images of neurospheres in suspension were taken under a microscope. (B and C) Transwell migration assay. GSLCs at a density of 5,000 cells/ml were plated in the top chamber of the Transwell insert and were treated with BIO or ICG-001 for $24 \mathrm{~h}$. Cells that migrated towards the lower chambers were fixed with methanol and stained with crystal violet. Cells were counted under a microscope in five random fields per well. Scale bars, $40 \mu \mathrm{m}$. (D and E) GSLCs were dissociated and seeded in suspension, after which they were treated with BIO or ICG-001. Then, the cells were processed with Annexin V-FITC and propidium iodide (PI) for analysis by flow cytometry (left). The Annexin V/PI-positive cells were counted as apoptotic cells. Fold-changes were calculated based on the data (right). Data represent the means \pm SD. ${ }^{*} \mathrm{P}<0.05$ different from the respective controls.

\section{Results}

Generation of GSLCs from U87 and U251 sphere cultures induces neural stem cell properties with the elevated expression of ALDHI and $\beta$-catenin. We enriched for primary neurospheres from the human glioma cell lines U87 and U251 by incubating the cells in neurobasal medium containing B27, bFGF and EGF for 7 days. Then, primary neurospheres were dissociated with Accutase into single cell suspensions. Primary neurospheres were incubated in neurobasal medium again to enrich for GSLCs (secondary neurospheres). GSLCs attached to the plate and began proliferating for 3 days after the serum-free medium was replaced with medium supplemented with serum (Fig. 1A). Our results suggest that GSLCs have the capacity for self-renewal and differentiation. As shown in Fig. 1B and $\mathrm{C}$, the protein expression level of $\beta$-catenin and CSC marker ALDH1 was significantly increased in U87 GSLCs and U251 GSLCs compared with U87 and U251 cells, respectively (Fig. 1B). A similar result was observed with immunofluorescence staining. ALDH1 (green fluorescence), which is located in the cell membrane, is upregulated in U87 GSLCs and U251 GSLCs compared with U87 and U251 cells, respectively (Fig. 1C). Blue fluorescence represents DAPI signal, which is located in the cell nucleus. These results show that U87 GSLCs and U251 GSLCs possess CSC characteristics relative to the parental cells, which includes elevated expression of ALDH1 and $\beta$-catenin.

The regulation of $\beta$-catenin activity is vital to maintain the neural stem cell traits of U87 GSLCs and U251 GSLCs. To determine whether the regulation of $\beta$-catenin activity is vital to maintain CSC properties, we activated $\beta$-catenin by treating U87 GSLCs and U251 GSLCs cells with the GSK3 $\beta$ inhibitor BIO $(2 \mu \mathrm{M})$ or we inactivated $\beta$-catenin/TCF transcription by treating U87 GSLCs and U251 GSLCs cells with ICG-001 $(10 \mu \mathrm{M})$. Our results showed that BIO treatment maintained sphere formation of U87 GSLCs and U251 GSLCs, whereas ICG-001 significantly decreased sphere formation of U87 GSLCs and U251 GSLCs (Fig. 2A). The Transwell migration assay was used to assess the effects of BIO and ICG-001 

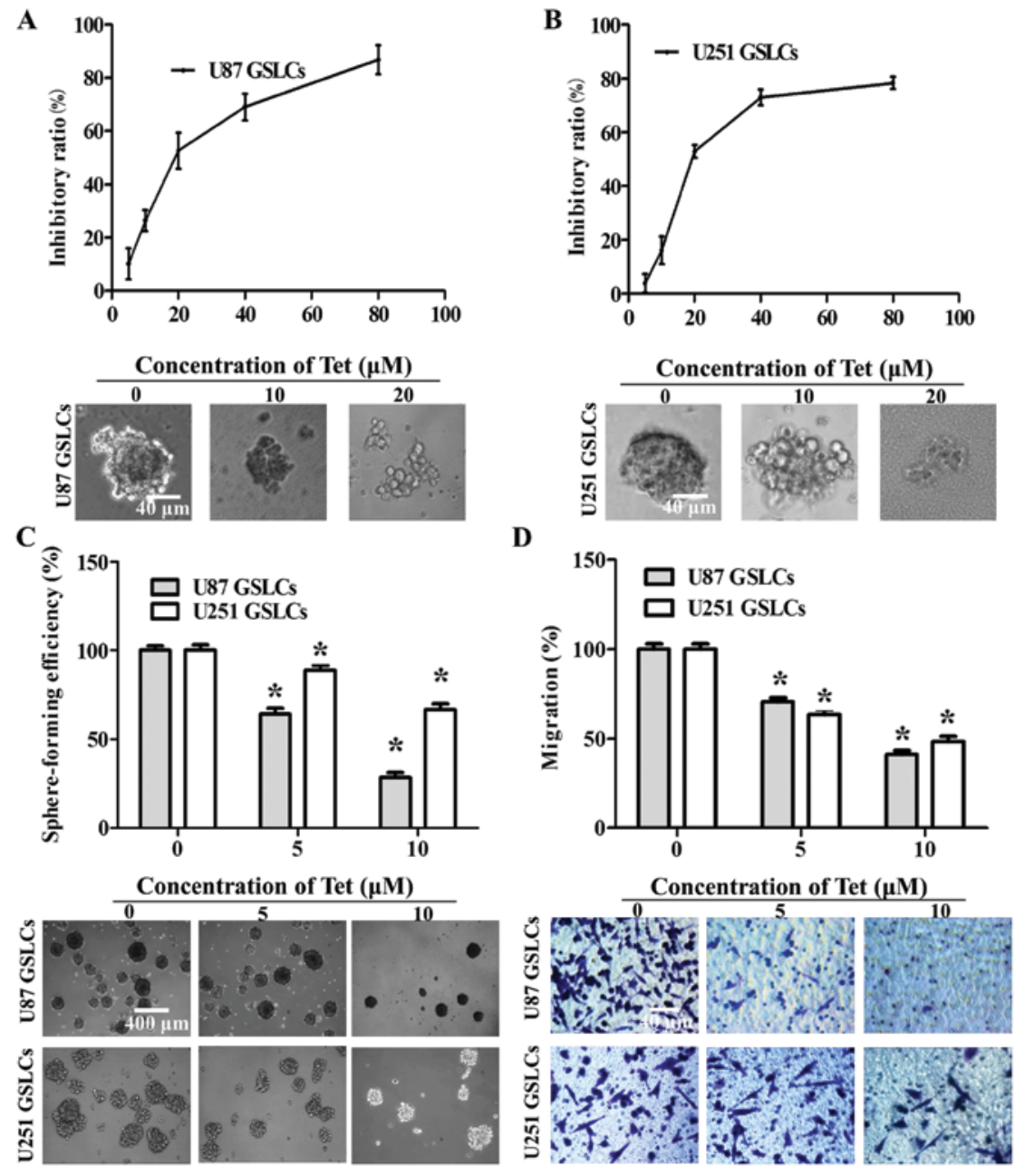

Figure 3. The inhibitory effects of Tet on U87 GSLCs and U251 GSLCs include reduced viability, neurosphere formation, and migration. (A and B) Cell viability was tested using a CCK-8 assay after treating U87 GSLCs and U251 GSLCs with various doses of Tet for 48 h (upper panel). GSLCs were dissociated and seeded in suspension culture, after which the cells were treated with Tet $(0-20 \mu \mathrm{M})$ for 7 days in 96 -well plates. Images of neurospheres in suspension were taken under a microscope (lower panel). Scale bars, $40 \mu \mathrm{m}$. (C) GSLCs were dissociated and seeded in suspension, after which they were treated with Tet $(0-10 \mu \mathrm{M})$ for $48 \mathrm{~h}$. Cells were then cultured in serum-free medium for 7 days to check sphere-forming efficiency. The number of GSLCs was counted under a microscope in five random fields per well. Scale bars, $400 \mu \mathrm{m}$. (D) Transwell migration assay. GSLCs were seeded at a density of 5,000 cells $/ \mathrm{ml} \mathrm{in}$ the top chamber of a Transwell insert, after which they were treated with Tet $(0-10 \mu \mathrm{M})$ for $24 \mathrm{~h}$. Cells that migrated to the lower chambers were fixed with methanol and stained with crystal violet. Cells were counted under a microscope in five random fields per well. Data represent the means $\pm \mathrm{SD}$. ${ }^{*} \mathrm{P}<0.05$ different from the respective controls. Scale bars, $400 \mu \mathrm{m}$.

on the migration of GSLCs in vitro. Our results show that compared with controls, the migration capability of GSLCs was significantly reduced following ICG-001 treatment, but the migration capability of GSLCs was conserved following BIO treatment (Fig. 2B and C). We next analyzed apoptosis of GSLCs upon treatment with BIO or ICG-001 by flow cytometry. The percentage of apoptotic GSLCs increased significantly after treatment with ICG-001, whereas GSLC apoptosis did not increase following BIO treatment (Fig. 2D and E). Our results suggest that the stem-like characteristics of U87 GSLCs and U251 GSLCs can be effectively inhibited by inactivating $\beta$-catenin signaling, and that activating $\beta$-catenin could promote the maintenance of CSC properties.

Tet inhibits cell viability, neurosphere formation, and the migration of U87 GSLCs and U251 GSLCs in vitro. To investigate whether Tet inhibits cell growth in GSLCs, we analyzed cell viability following Tet treatment using a CCK-8 assay. U87 GSLCs and U251 GSLCs were grown in neurobasal medium containing B27, bFGF and EGF in the presence of various doses of Tet $(0-80 \mu \mathrm{M})$. The spheroids in each well were photographed at the end of the incubation period. Our results show that Tet treatment decreases the viability of GSLCs in a dose-dependent manner (Fig. $3 \mathrm{~A}$ and $\mathrm{B}$ ). Tet $\mathrm{IC}_{50}$ values in U87 GSLCs and U251 GSLCs were 30.41 and $27.5 \mu \mathrm{M}$, respectively. When GSLCs were dissociated, suspended, and treated with Tet $(0-10 \mu \mathrm{M})$ for 7 days, we observed changes in cell morphology. Tet treatment decreased sphere formation in GSLCs in a dose-dependent manner (Fig. 3C), suggesting that Tet may inhibit the self-renewal capacity of GSLCs. Additionally, a Transwell migration assay was used to investigate the effects of Tet on the migration of GSLCs in vitro. Tet treatment reduced the migration efficiency of GSLCs in a dose-dependent manner (Fig. 3D).

Tet induces the apoptosis of GSLCs as indicated by the upregulation of Bax, the cleavage of PARP, and the downregulation of $B c l-2$. To understand the mechanisms underlying the effects 
A

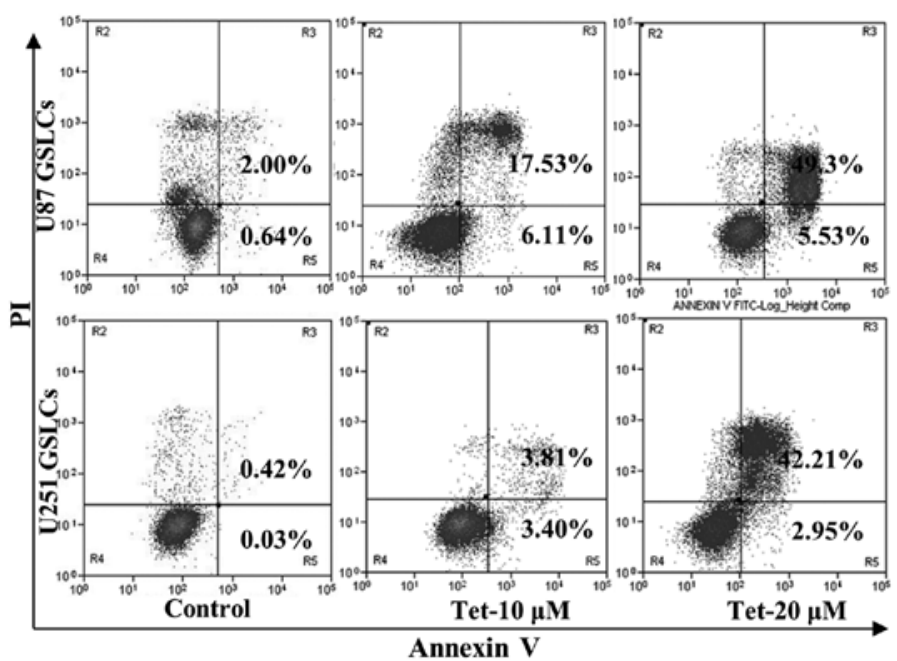

B

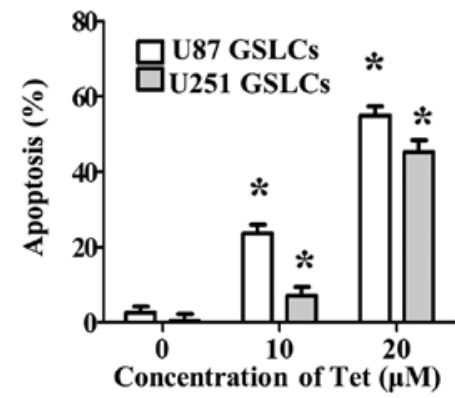

C

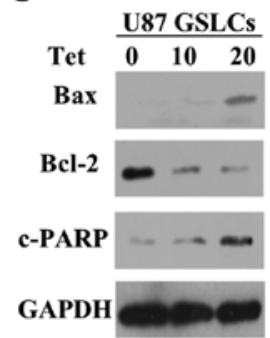

D

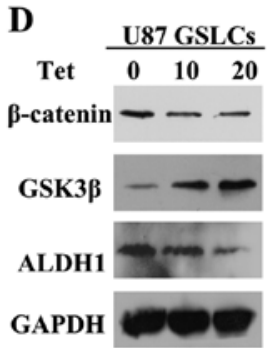

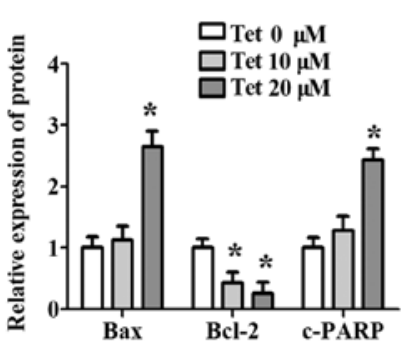

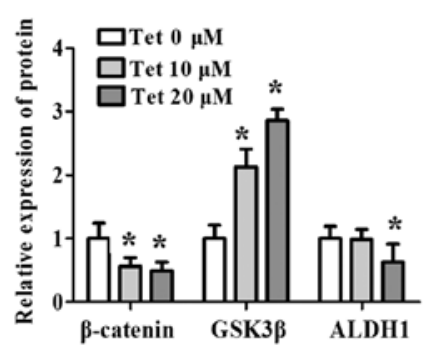

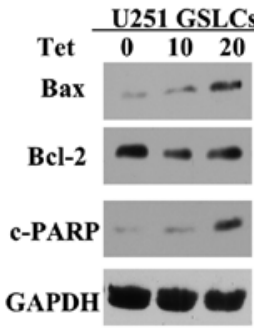
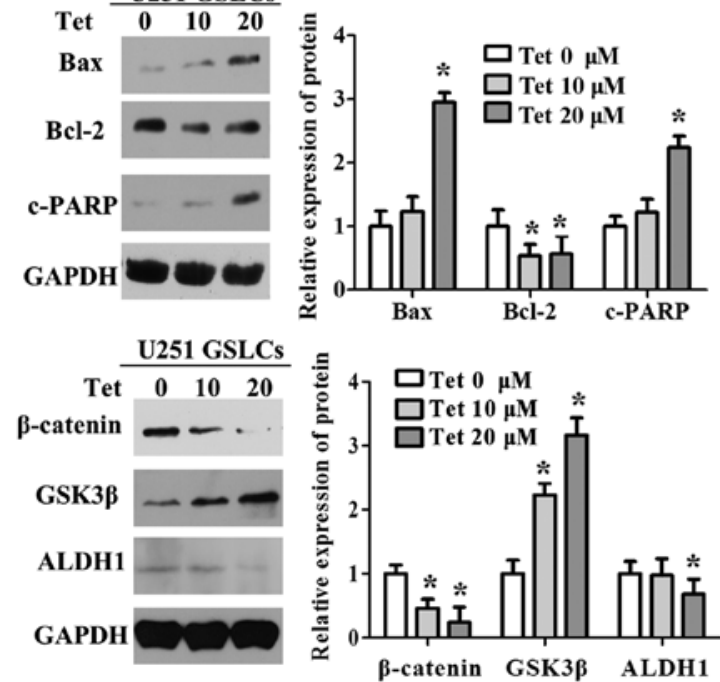

Figure 4. Tet induces GSLC apoptosis, upregulates expression of GSK3 $\beta$, Bax and cleaves PARP, and downregulates expression of $\beta$-catenin, Bcl-2 and ALDH1. (A and B) GSLCs were dissociated, seeded in suspension and treated with Tet for $48 \mathrm{~h}$. The cells were then stained with Annexin V-FITC and propidium iodide (PI) and analyzed by flow cytometry (left). The Annexin V/PI-positive cells were considered apoptotic cells. Fold-changes were calculated (right). (C and D) GSLCs were treated with Tet $(0-20 \mu \mathrm{M})$ for $48 \mathrm{~h}$. The expression levels of Bcl-2, Bax, c-PARP, $\beta$-catenin, GSK3 $\beta$, ALDH1 and GAPDH were determined by western blot analysis (left). Fold-changes were calculated (right). Data represent the means $\pm \mathrm{SD}$. " $\mathrm{P}<0.05$ different from the respective controls.

of Tet on U87 GSLCs and U251 GSLCs, we analyzed the rate of apoptosis in GSLCs treated with Tet by flow cytometry. Our results show that the proportion of apoptotic GSLCs increases in a dose-dependent manner in response to Tet treatment (Fig. 4A and B). We next assessed whether GSLC treatment with Tet has an effect on the expression of apoptosis-related proteins, including Bax, Bcl-2 and cleaved PARP. We found that Tet treatment results in significant downregulation of the expression of the anti-apoptotic protein Bcl-2, upregulation of the expression of the apoptosis-promoting protein Bax, and an increase in the cleavage of PARP in U87 GSLCs and U251 GSLCs (Fig. 4C).

Tet inhibits neural stem cell properties of GSLCs with the upregulation of GSK3 $\beta$ and the upregulation of $\beta$-catenin. We next analyzed whether Tet has an effect on the Wnt/ $\beta$-catenin pathway as well as the stemness marker ALDH1. Upon dissociation, suspension and treatment of GSLCs with Tet for $48 \mathrm{~h}$, we found that Tet significantly reduced the protein expression levels of $\beta$-catenin and increased the expression of GSK3 $\beta$. Moreover, the stem cell marker ALDH1 was significantly downregulated in response to treatment with $20 \mu \mathrm{M}$ Tet (Fig. 4D). These data indicate that Tet has inhibitory effects on GSLCs, which are partly associated with the repression of the Wnt $/ \beta$-catenin pathway.

Tet inhibits GSLCs by repressing the nuclear translocation and expression of $\beta$-catenin. To determine whether the inhibitory effects of Tet are related to the downregulation and inactivation of $\beta$-catenin, we assessed additional Wnt pathway-related proteins ( $\beta$-catenin and GSK3 $\beta$ ), the CSC marker ALDH1, and apoptosis-related proteins (Bcl-2, Bax and c-PARP) upon treatment of GSLCs with $2 \mu \mathrm{M}$ BIO, $10 \mu \mathrm{M}$ ICG-001, or $20 \mu \mathrm{M}$ Tet treatment (Fig. 5A-D). The expression levels of GSK3 $\beta$, Bax and cleaved PARP significantly increased, whereas the expression levels of $\beta$-catenin, ALDH1 and Bcl-2 significantly decreased following ICG-001 and Tet treatment. Compared with Tet treatment, BIO treatment 
$\mathbf{A}$

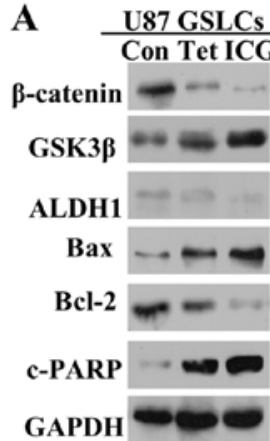

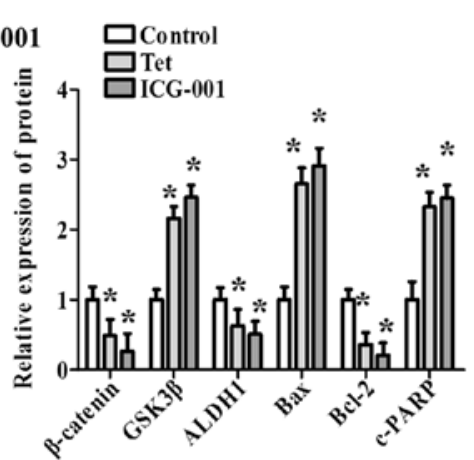
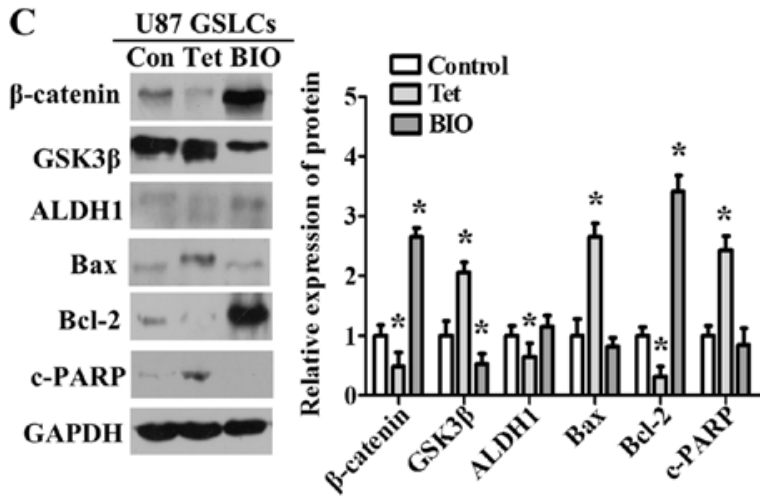

$\mathbf{E}$

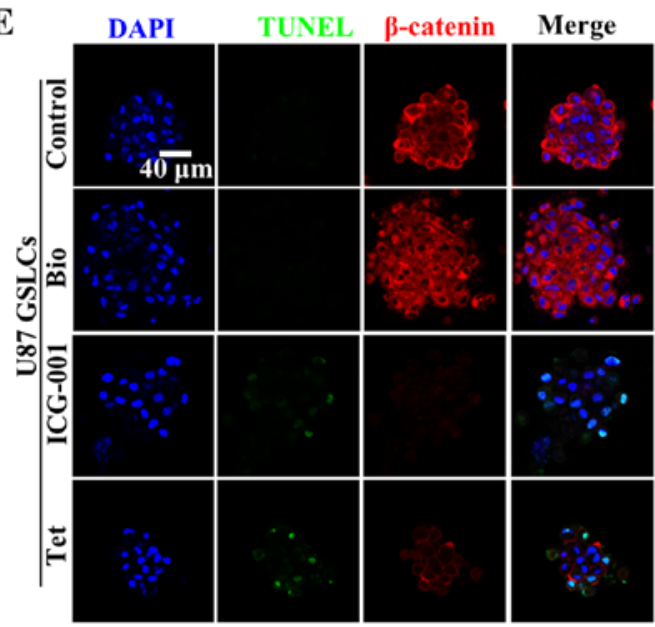

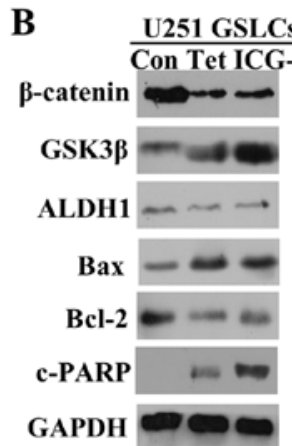

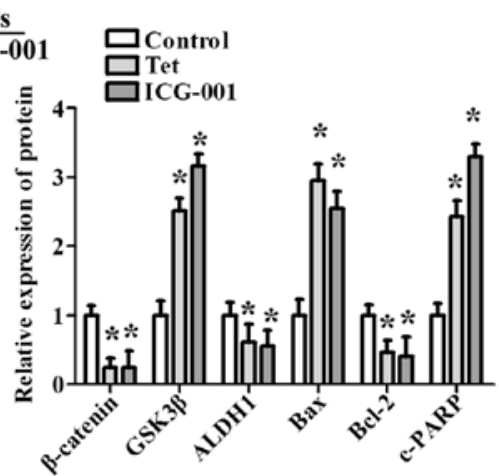

D
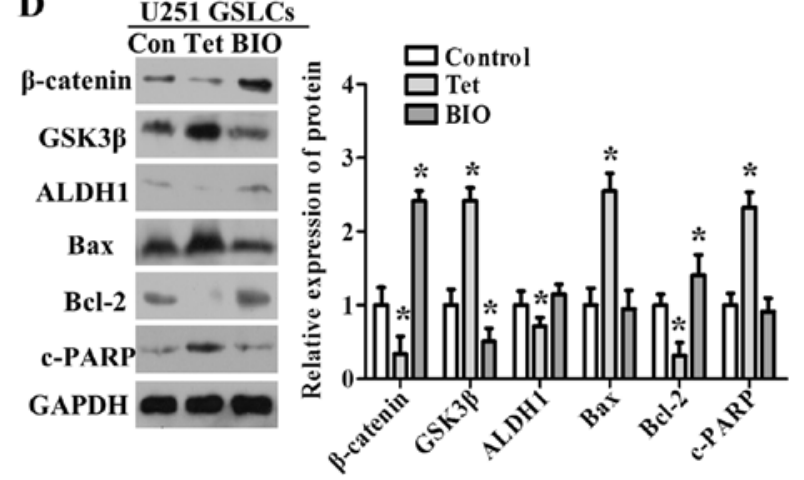

$\mathbf{F}$

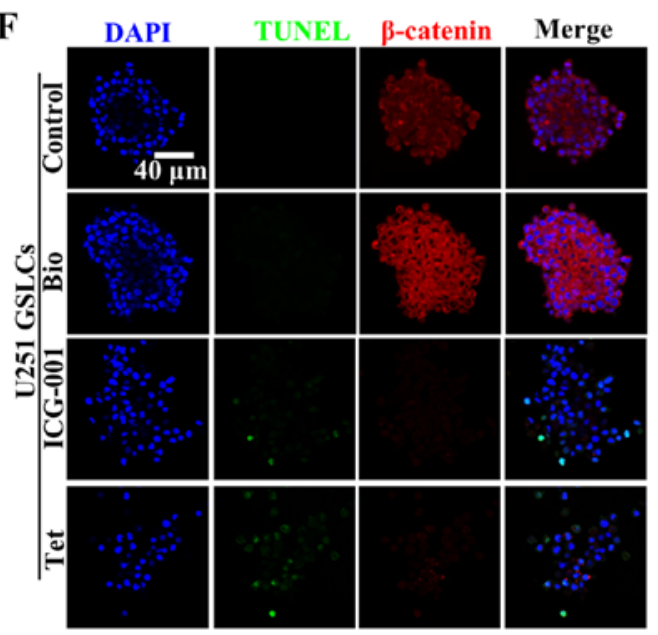

Figure 5. Tet inhibits $\beta$-catenin expression and nuclear translocation to modulate the Wnt signaling pathway and stimulates apoptosis in U87 GSLCs and U251 GSLCs. (A-D) GSLCs were dissociated and seeded in suspension culture, after which they were treated with Tet or BIO (A and B) or Tet or ICG-001 (C and D). The expression levels of $\beta$-catenin, GSK3 $\beta$, ALDH1, Bcl-2, Bax, c-PARP and GAPDH were determined by western blot analysis (left panel). Fold-changes were calculated (right panel). The data represent the means $\pm \mathrm{SD}$. ${ }^{*} \mathrm{P}<0.05$ different from the respective controls. (E and F) Confocal immunofluorescence staining. U87 GSLCs and U251 GSLCs were seeded in suspension and treated with control, Tet, BIO or ICG-001 for 48 h. Blue fluorescence represents DAPI, red fluorescence represents $\beta$-catenin, and green fluorescence represents TUNEL. Scale bars, $40 \mu \mathrm{m}$.

downregulated the expression of GSK3 $\beta$ and upregulated the expression of $\beta$-catenin and Bcl-2. We further analyzed the proportion of apoptotic cells and the expression and localization of $\beta$-catenin by TUNEL assay and confocal immunofluorescence staining, respectively, following treatment with BIO, ICG-001 and Tet (Fig. 5E and F). Our results show that $\beta$-catenin becomes concentrated in the cytoplasm and nucleus, and TUNEL staining is negative in response to BIO treatment. Upon ICG-001 or Tet treatment, the expression of $\beta$-catenin decreased and TUNEL staining increased. These results suggest that the nuclear translocation and expression of $\beta$-catenin are vital for cell survival and the maintenance of CSC properties in U87 GSLCs and U251 GSLCs.
Tet inhibits the $\beta$-catenin activation induced by BIO. To determine whether Tet inhibited the $\beta$-catenin signaling activation induced by $\mathrm{BIO}$, we assessed nuclear $\beta$-catenin expression upon treatment of GSLCs with $20 \mu \mathrm{M}$ Tet or $20 \mu \mathrm{M}$ Tet $+2 \mu \mathrm{M}$ BIO treatment. The expression levels of nuclear $\beta$-catenin significantly decreased following Tet and Tet $+\mathrm{BIO}$ treatment. Compared with the control treatment group, the Tet + BIO treatment group downregulated the expression of nuclear $\beta$-catenin significantly (Fig. 6).

Association between $\beta$-catenin nuclear localization and clinicopathological features. Immunohistochemical detection of $\beta$-catenin was analyzed in 88 gliomas for which sufficient 

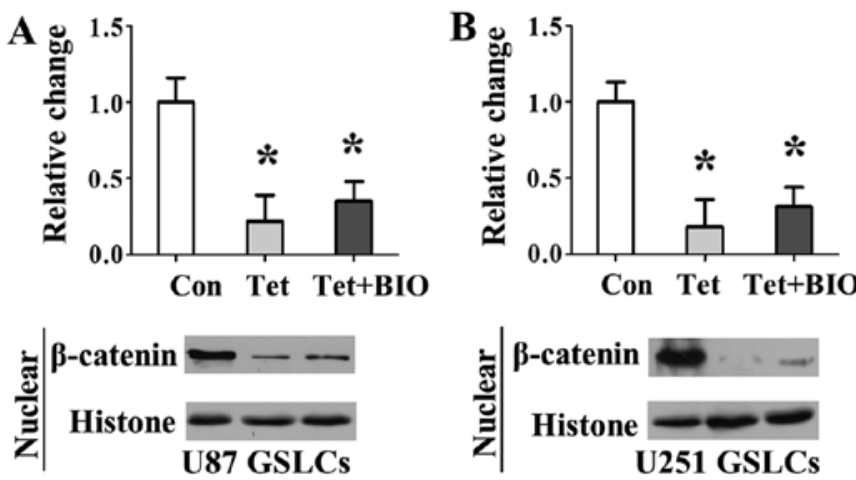

Figure 6. Tet inhibits the $\beta$-catenin activation induced by BIO. (A and B) GSLCs were dissociated and seeded in suspension culture, after which they were treated with $20 \mu \mathrm{M}$ Tet or $2 \mu \mathrm{M}$ BIO $+20 \mu \mathrm{M}$ Tet treatment. Nuclear proteins were prepared by using a protein extraction kit (Beyotime Institute of Biotechnology, Haimen, China) according to the manufacturer's protocol. The expression of nuclear $\beta$-catenin was determined by western blot analysis. Fold-changes were calculated. The data represent the means $\pm \mathrm{SD}$. ${ }^{*} \mathrm{P}<0.05$ different from the respective controls.

$\mathbf{A}$

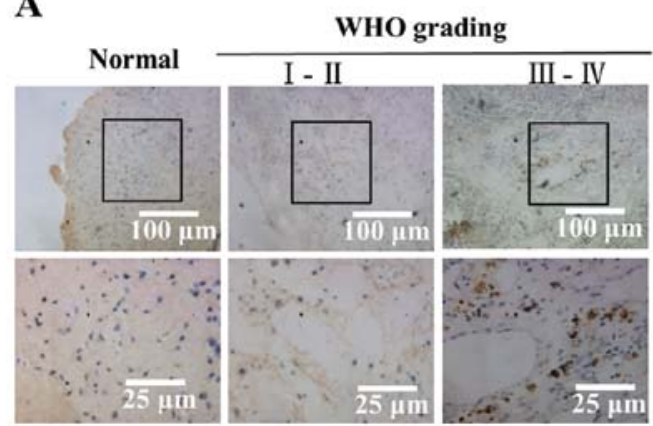

B

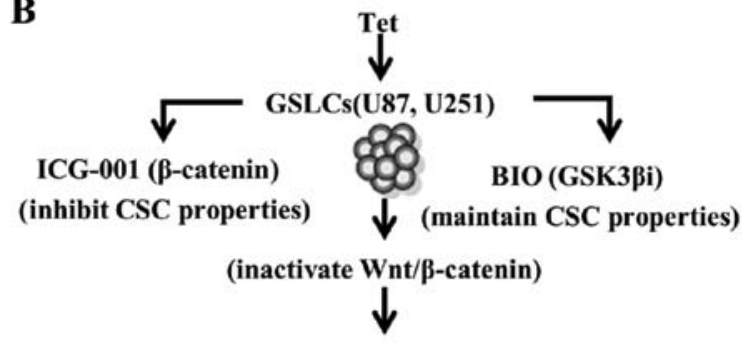

(Cell viability, sphere formation, migration and Apoptosis) ( $\beta$-catenin, GSK3 $\beta$, ALDH1, Bax, Bcl-2, c-PARP and TUNEL)

Figure 7. Different expression levels of $\beta$-catenin in normal brain tissue and different grades of glioma and a schematic overview of the anticancer action of Tet. (A) Immunohistochemical staining of $\beta$-catenin in glioma specimens and normal brain tissue. Scale bars, $100 \mu \mathrm{m}$ (left), $25 \mu \mathrm{m}$ (right). (B) Schematic overview of the anticancer action of Tet.

Table I. Correlation between the expression of $\beta$-catenin and clinicopathological parameters in glioma patients $\left(\chi^{2}\right.$ test).

\begin{tabular}{lccccc}
\hline \multicolumn{5}{c}{} & \multicolumn{3}{c}{$\beta$-catenin expression } & & \\
Variables & $\mathrm{n}$ & $\begin{array}{c}\text { Cytoplasm/ } \\
\text { member }\end{array}$ & Nuclear & $\chi^{2}$ & P-value \\
\hline Gender & & & & 2.503 & 0.114 \\
$\quad$ Male & 51 & 23 & 28 & & \\
Female & 37 & 23 & 14 & & \\
Age (years) & & & & 0.059 & 0.808 \\
$<36$ & 41 & 22 & 19 & & \\
$\geq 36$ & 47 & 24 & 23 & & \\
WHO grading & & & & 43.591 & $\mathbf{0 . 0 0 1}$ \\
I-II & 47 & 40 & 7 & & \\
III-IV & 41 & 6 & 35 & & \\
\hline
\end{tabular}

Significant P-values are shown in bold.

tissue was available. WHO grading for the samples was distributed as follows: I + II, 47 cases; III + IV, 41 cases. According to our IHC staining results, in tumor cells, $\beta$-catenin signal was observed in the cytoplasm, nucleus, or both, and the $\beta$-catenin levels were heterogeneous. In contrast, $\beta$-catenin staining in normal brain tissue was constantly negative (Fig. 7A). As shown in Table I, $\beta$-catenin immunoreactivity in the nucleus was low in grade I-II tumors, whereas $\beta$-catenin signal increased significantly in grade III-IV tumors. Nuclear $\beta$-catenin immunoreactivity significantly correlated with WHO grading based on analysis using the chi-square test $\left(\chi^{2}=43.59, P=0.001\right)$. No significant differences between gender and age were observed. These results suggest that the nuclear expression of $\beta$-catenin is related to the degree of malignancy in gliomas.

\section{Discussion}

Recent studies have shown that cancer stem cells in malignant glioma closely correlate with poor prognosis. Glioma patients often relapse after treatment because of the persistence of glioma stem cells. In the present study, we enriched cells with CSC properties to obtain U87 GSLCs and U251 GSLCs based on high levels of ALDH1 expression. Tet cytotoxicity was demonstrated in U87 GSLCs and U251 GSLCs. Importantly, as shown in Fig. 7B, we found for the first time that Tet induces GSLC apoptosis by repressing the expression of $\beta$-catenin and preventing $\beta$-catenin nuclear translocation.

A small fraction of cancer stem cells (CSC) are thought to initiate cancer and drive the tumorigenesis of gliomas. Studies have shown that ALDH1 activity can be used as a marker to 
identify stem-like cells in glioma, breast, liver, hepatic cancer and embryonal rhabdomyosarcoma (16-20). We enriched for GSLCs from glioma cell lines U87 and U251 using a neurosphere culture technique, and we resuspended the GSLCs for subculture using Accutase. The data in Fig. 1B and C show that ALDH1 is highly expressed in U87 GSLCs and U251 GSLCs compared with their parental cell lines. This suggests that we enriched U87 GSLCs and U251 GSLCs based on increased expression of the cancer stem cell marker ALDH1.

The Wnt/ $\beta$-catenin pathway is one of the key signaling pathways in cancer stem cells. A recent study also showed that the suppression of GSK3 $\beta$ activity enhances $\beta$-catenin signaling and increases the level of nuclear $\beta$-catenin, which promotes the stemness of colorectal cancer cells (21). The inactivation of $\mathrm{Wnt} / \beta$-catenin signaling results in decreased tumor growth and increased invasive ability of glioma stem cells $(8,22)$. The activation of $\mathrm{Wnt} / \beta$-catenin signaling contributes to the maintenance of glioma stem cells and the stem cell phenotype in glioma $(7,23,24)$. Our results show that the pharmacologic inhibition of GSK $3 \beta$ by BIO treatment in U87 GSLCs and U251 GSLCs results in increased $\beta$-catenin activity as well as no change in sphere formation, migration capability, or apoptosis compared with control cells. In contrast, the pharmacologic inhibition of $\beta$-catenin in U87 GSLCs and U251 GSLCs by ICG-001, which decreases the expression of nuclear $\beta$-catenin and $\beta$-catenin transcription, resulted in reduced sphere formation and migration capability and an increase in apoptosis compared with control cells (Fig. 2). In addition, the apoptosis-related protein Bcl-2 was upregulated and c-PARP was decreased in GSLCs in response to $\mathrm{BIO}$ treatment, whereas $\mathrm{Bcl}-2$ was downregulated, and Bax and c-PARP were upregulated in GSLCs in response to ICG-001 treatment (Fig. 4).

Recent studies have reported that Tet is a strong anticancer agent based on in vitro and in vivo studies (25). Tet also showed a time- and concentration-dependent cytotoxic effect on neuroblastoma cells (11). In this study, we found for the first time that Tet reduces the viability, neurosphere formation capacity, and migration capacity in GSLCs in a dose-dependent manner (Fig. 3). Tet treatment also induced apoptosis in GSLCs by reducing the expression levels of $\beta$-catenin, increasing the protein expression of GSK $3 \beta$, inactivating the anti-apoptotic protein $\mathrm{Bcl}-2$, upregulating the apoptosis-promoting protein Bax, and promoting the cleavage of PARP (Fig. 4). The Wnt/ $\beta$-catenin signaling pathway is associated with the anticancer activity of Tet in chronic myeloid leukemia and human colorectal cancer, which is consistent with our results $(26,27)$. Moreover, our results are in agreement with the finding that Tet treatment reduces Bcl-2 levels, increases Bax levels, and promotes the cleavage of PARP in other cancer cells (28-30). These data suggest that Tet may induce GSLC apoptosis partly through the inhibition of the $\mathrm{Wnt} / \beta$-catenin signaling pathway.

Wnt $/ \beta$-catenin signaling plays a critical role in cancer cell proliferation and invasion. $\beta$-catenin protein is persistently upregulated in a variety of human cancers, including glioma (31). The nuclear translocation and expression of $\beta$-catenin is critical for the survival, invasion and tumorigenesis of glioma (32-34). Previous studies have reported that aberrant $\mathrm{Wnt} / \beta$-catenin signaling is essential for the maintenance of cancer stem cells (CSCs) of various origins, including the bladder, blood, breast and colon (35). Our results suggest that the inhibition of $\mathrm{Wnt} / \beta$-catenin signaling in CSCs could be an effective treatment for cancer, which has been suggested by other studies (36). The Wnt/ $\beta$-catenin pathway critically regulates the self-renewal and differentiation of neural stem/ progenitor cells (37-39). A previous study showed that the inhibition of nuclear $\beta$-catenin expression decreases the in vitro proliferation and sphere formation capability of glioma stem cells (40). These results suggest that $\mathrm{Wnt} / \beta$-catenin signaling activation is vital for the survival of glioma stem cells. GSCs are also closely related to the degree of malignancy in gliomas. In this study, we found that the nuclear expression of $\beta$-catenin in glioma tissues significantly correlated with WHO grading (Fig. 6A). Tet treatment inhibits GSLCs by repressing the nuclear translocation and expression of $\beta$-catenin (Fig. 5A-E). Tet treatment (10 mg/kg body weight) also inhibits tumor metastasis in the mouse breast in vivo (12). Additional studies in other cell lines and animal models will be necessary to demonstrate the inhibitory effect of Tet on GSLCs through the repression of $\beta$-catenin signaling.

In conclusion, we observed for the first time that Tet inhibits cell viability, neurosphere formation, and migration in U87 GSLCs and U251 GSLCs in vitro. The WHO grading of glioma tissues was significantly correlated with the nuclear expression of $\beta$-catenin. Importantly, Tet treatment repressed the nuclear translocation and expression of $\beta$-catenin and induced apoptosis in GSLCs through the upregulation of Bax, the cleavage of PARP, and the downregulation of Bcl-2. Our results provide a basis for Tet development and provide novel insight into GSC-based anti-glioma treatments.

\section{Acknowledgements}

The present study was supported by the Science and Technology Project of Guangzhou (no. 2014J4100103), the China Postdoctoral Science Foundation (no. 2015M572414), the Guangdong Natural Science Foundation (no. 2016A030310096) and the Shenzhen Science and Technology Project (no. JCYJ20150324141711568).

\section{References}

1. Parsons DW, Jones S, Zhang X, Lin JC, Leary RJ, Angenendt P, Mankoo P, Carter H, Siu IM, Gallia GL, et al: An integrated genomic analysis of human glioblastoma multiforme. Science 321: 1807-1812, 2008.

2. You G, Sha ZY, Yan W, Zhang W, Wang YZ, Li SW, Sang L, Wang Z, Li GL, Li SW, et al: Seizure characteristics and outcomes in 508 Chinese adult patients undergoing primary resection of low-grade gliomas: A clinicopathological study. Neuro Oncol 14: 230-241, 2012.

3. Dahlrot RH: The prognostic value of clinical factors and cancer stem cell-related markers in gliomas. Dan Med J 61: B4944, 2014.

4. Tabatabai G and Weller M: Glioblastoma stem cells. Cell Tissue Res 343: 459-465, 2011.

5. D'Uva G, Bertoni S, Lauriola M, De Carolis S, Pacilli A, D'Anello L, Santini D, Taffurelli M, Ceccarelli C, Yarden Y, et al: Beta-catenin/HuR post-transcriptional machinery governs cancer stem cell features in response to hypoxia. PLoS One 8: e80742, 2013.

6. Korkaya H, Kim GI, Davis A, Malik F, Henry NL, Ithimakin S, Quraishi AA, Tawakkol N, D'Angelo R, Paulson AK, et al: Activation of an IL6 inflammatory loop mediates trastuzumab resistance in HER2 ${ }^{+}$breast cancer by expanding the cancer stem cell population. Mol Cell 47: 570-584, 2012. 
7. Chen X, Hu W, Xie B, Gao H, Xu C and Chen J: Downregulation of SCAI enhances glioma cell invasion and stem cell like phenotype by activating Wnt/ $\beta$-catenin signaling. Biochem Biophys Res Commun 448: 206-211, 2014.

8. Rathod SS, Rani SB, Khan M, Muzumdar D and Shiras A: Tumor suppressive miRNA-34a suppresses cell proliferation and tumor growth of glioma stem cells by targeting Akt and Wnt signaling pathways. FEBS Open Bio 4: 485-495, 2014.

9. Brabletz S, Schmalhofer O and Brabletz T: Gastrointestinal stem cells in development and cancer. J Pathol 217: 307-317, 2009.

10. Wang $\mathrm{H}$ and Chen $\mathrm{X}$ : Tetrandrine ameliorates cirrhosis and portal hypertension by inhibiting nitric oxide in cirrhotic rats. J Huazhong Univ Sci Technolog Med Sci 24: 385-388, 395, 2004.

11. Chen Y, Chen JC and Tseng SH: Effects of tetrandrine plus radiation on neuroblastoma cells. Anticancer Res 29: 3163-3171, 2009.

12. Gao JL, Ji X, He TC, Zhang Q, He K, Zhao Y, Chen SH and Lv GY: Tetrandrine suppresses cancer angiogenesis and metastasis in 4T1 tumor bearing mice. Evid Based Complement Alternat Med 2013: 265061, 2013

13. Cho HS, Chang SH, Chung YS, Shin JY, Park SJ, Lee ES, Hwang SK, Kwon JT, Tehrani AM, Woo M, et al: Synergistic effect of ERK inhibition on tetrandrine-induced apoptosis in A549 human lung carcinoma cells. J Vet Sci 10: 23-28, 2009.

14. Wu K, Zhou M, Wu QX, Yuan SX, Wang DX, Jin JL, Huang J, Yang JQ, Sun WJ, Wan LH, et al: The role of IGFBP-5 in mediating the anti-proliferation effect of tetrandrine in human colon cancer cells. Int J Oncol 46: 1205-1213, 2015.

15. Kou B, Liu W, He W, Zhang Y, Zheng J, Yan Y, Zhang Y, Xu S and Wang H: Tetrandrine suppresses metastatic phenotype of prostate cancer cells by regulating Akt/mTOR/MMP-9 signaling pathway. Oncol Rep 35: 2880-2886, 2016.

16. Kida K, Ishikawa T, Yamada A, Shimada K, Narui K, Sugae S, Shimizu D, Tanabe M, Sasaki T, Ichikawa Y, et al: Effect of ALDH1 on prognosis and chemoresistance by breast cancer subtype. Breast Cancer Res Treat 156: 261-269, 2016.

17. Gehlot P, Shukla V, Gupta S and Makidon PE: Detection of ALDH1 activity in rabbit hepatic VX2 tumors and isolation of ALDH1 positive cancer stem cells. J Transl Med 14: 49, 2016.

18. Zhang Y, Wang SX, Ma JW, Li HY, Ye JC, Xie SM, Du B and Zhong XY: EGCG inhibits properties of glioma stem-like cells and synergizes with temozolomide through downregulation of P-glycoprotein inhibition. J Neurooncol 121: 41-52, 2015

19. Nakahata K, Uehara S, Nishikawa S, Kawatsu M, Zenitani M, Oue T and Okuyama H: Aldehyde dehydrogenase 1 (ALDH1) is a potential marker for cancer stem cells in embryonal rhabdomyosarcoma. PLoS One 10: e0125454, 2015.

20. Liu M, Inoue K, Leng T, Guo S and Xiong ZG: TRPM7 channels regulate glioma stem cell through STAT3 and Notch signaling pathways. Cell Signal 26: 2773-2781, 2014.

21. Venugopal A, Subramaniam D, Balmaceda J, Roy B, Dixon DA Umar S, Weir SJ and Anant S: RNA binding protein RBM3 increases beta-catenin signaling to increase stem cell characteristics in colorectal cancer cells. Mol Carcinog 55: 1503-1516, 2015.

22. Gong A and Huang S: FoxM1 and Wnt $/ \beta$-catenin signaling in glioma stem cells. Cancer Res 72: 5658-5662, 2012.

23. Kim KH, Seol HJ, Kim EH, Rheey J, Jin HJ, Lee Y, Joo KM, Lee $J$ and Nam DH: Wnt/ $\beta$-catenin signaling is a key downstream mediator of MET signaling in glioblastoma stem cells. Neuro Oncol 15: 161-171, 2013.
24. Shi L, Fei X, Wang Z and You Y: PI3K inhibitor combined with miR-125b inhibitor sensitize TMZ-induced anti-glioma stem cancer effects through inactivation of Wnt/beta-catenin signaling pathway. In Vitro Cell Dev Biol Anim 51: 1047-1055, 2015.

25. Bhagya N and Chandrashekar KR: Tetrandrine - A molecule of wide bioactivity. Phytochemistry 125: 5-13, 2016.

26. Xu XH, Gan YC, Xu GB, Chen T, Zhou H, Tang JF, Gu Y, Xu F, Xie YY, Zhao XY, et al: Tetrandrine citrate eliminates imatinibresistant chronic myeloid leukemia cells in vitro and in vivo by inhibiting Bcr-Abl/ $\beta$-catenin axis. J Zhejiang Univ Sci B 13: 867-874, 2012

27. He BC, Gao JL, Zhang BQ, Luo Q, Shi Q, Kim SH, Huang E, Gao Y, Yang K, Wagner ER, et al: Tetrandrine inhibits Wnt//3catenin signaling and suppresses tumor growth of human colorectal cancer. Mol Pharmacol 79: 211-219, 2011.

28. Zhang YX, Liu XM, Wang J, Li J, Liu Y, Zhang H, Yu XW and Wei N: Inhibition of AKT/FoxO3a signaling induced PUMA expression in response to p53-independent cytotoxic effects of H1: A derivative of tetrandrine. Cancer Biol Ther 16: 965-975, 2015.

29. Zhu R, Liu T, Tan Z, Wu X, Li M, Jiang L, Bao R, Shu Y, Lu A and Liu Y: Tetrandrine induces apoptosis in gallbladder carcinoma in vitro. Int J Clin Pharmacol Ther 52: 900-905, 2014.

30. Yu VW and Ho WS: Tetrandrine inhibits hepatocellular carcinoma cell growth through the caspase pathway and G2/M phase. Oncol Rep 29: 2205-2210, 2013.

31. Zheng H, Ying H, Wiedemeyer R, Yan H, Quayle SN, Ivanova EV, Paik JH, Zhang H, Xiao Y, Perry SR, et al: PLAGL2 regulates Wnt signaling to impede differentiation in neural stem cells and gliomas. Cancer Cell 17: 497-509, 2010.

32. Náger M, Santacana M, Bhardwaj D, Valls J, Ferrer I, Nogués P, Cantí C and Herreros J: Nuclear phosphorylated Y142 $\beta$-catenin accumulates in astrocytomas and glioblastomas and regulates cell invasion. Cell Cycle 14: 3644-3655, 2015.

33. Kahlert UD, Suwala AK, Koch K, Natsumeda M, Orr BA, Hayashi M, Maciaczyk J and Eberhart CG: Pharmacologic Wnt inhibition reduces proliferation, survival, and clonogenicity of glioblastoma cells. J Neuropathol Exp Neurol 74: 889-900, 2015.

34. Zhang N, Wei P, Gong A, Chiu WT, Lee HT, Colman H, Huang H, Xue J, Liu M, Wang Y, et al: FoxM1 promotes $\beta$-catenin nuclear localization and controls Wnt target-gene expression and glioma tumorigenesis. Cancer Cell 20: 427-442, 2011.

35. Holland JD, Klaus A, Garratt AN and Birchmeier W: Wnt signaling in stem and cancer stem cells. Curr Opin Cell Biol 25: 254-264, 2013

36. Takebe N, Harris PJ, Warren RQ and Ivy SP: Targeting cancer stem cells by inhibiting Wnt, Notch, and Hedgehog pathways. Nat Rev Clin Oncol 8: 97-106, 2011.

37. Chenn A and Walsh CA: Regulation of cerebral cortical size by control of cell cycle exit in neural precursors. Science 297: 365-369, 2002.

38. Clevers H, Loh KM and Nusse R: Stem cell signaling. An integral program for tissue renewal and regeneration: Wnt signaling and stem cell control. Science 346: 1248012, 2014.

39. Nusse R: Wnt signaling and stem cell control. Cell Res 18: 523-527, 2008.

40. Kierulf-Vieira KS, Sandberg CJ, Grieg Z, Günther CC, Langmoen IA and Vik-Mo EO: Wnt inhibition is dysregulated in gliomas and its re-establishment inhibits proliferation and tumor sphere formation. Exp Cell Res 340: 53-61, 2016. 